\title{
L'everolimus nella pratica clinica del trapianto di rene
}

\author{
E. Bertoni, M. Salvadori
}

Nefrologia e Nefrologia dei Trapianti, Azienda Ospedaliero-Universitaria, Careggi, Firenze

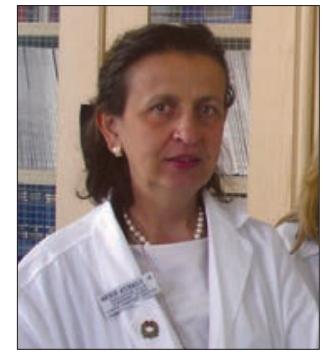

Elisabetta Bertoni
La progressiva evoluzione delle terapie immunosoppressive nel trapianto di rene ha portato a una riduzione dei rigetti acuti e a un netto miglioramento della sopravvivenza d'organo a breve termine. Questo soprattutto grazie all'impiego degli inibitori delle calcineurine $(\mathrm{CNI})$ che rappresentano tuttora la pietra miliare dell'immunosoppressione nel trapianto d'organo.

Tuttavia l'impiego di CNI è associato a lungo termine a nefrotossicità e ad altri effetti avversi come ipertensione, dislipidemia, diabete: tutti fattori che possono favorire la disfunzione cronica del trapianto. Anche per effetto di ciò, la sopravvivenza a lungo termine del trapianto non si è sostanzialmente modificata, come ci si attendeva (1).

Recentemente questo fatto ha portato a trovare nuovi schemi immunosoppressivi che permettano di ridurre i dosaggi di CNI o addirittura di sospenderli completamente. In quest'ambito l'everolimus gioca un ruolo centrale.

L'everolimus appartiene a un gruppo di farmaci (inibitori delle mTOR (mammalian target of rapamycin)), il cui capostipite è il sirolimus. Il sirolimus, scoperto nel 1964 nel terreno dell'isola di Pasqua, è un lattone macrociclico dotato di proprietà antibatteriche isolato dal fungo Streptomyces Hygroscopius. Ha poi rivelato importanti attività immunosoppressive (2).

L'everolimus è un nuovo macrolide di sintesi che ha rivelato importanti attività immunosoppressive nel trapianto di rene, cuore, polmone e fegato. Everolimus, ha maggiore polarità ed è più idrofilico del sirolimus, ed è stato sviluppato per migliorare la farmacocinetica e la farmacodinamica del sirolimus. Infatti, nonostante la simile struttura chimica, everolimus ha una più elevata biodisponibilità quando somministrato per via orale (20\% vs $14 \%$ ) ed ha un minore legame con le proteine
(74\% vs $92 \%$ ). Questo permette all'everolimus di avere un minor tempo per raggiungere lo steady state (4 giorni vs 7 giorni). Di conseguenza il sirolimus necessita all'inizio di un carico orale maggiore che può facilitare l'insorgenza di alcuni effetti clinici indesiderati. Inoltre la ridotta semivita dell'everolimus facilita aggiustamenti posologici, utili soprattutto quando è somministrato insieme a CNI per evitare la nefrotossicità. Le principali caratteristiche di farmacocinetica sono riassunte nella Tabella I (3).

Come il sirolimus, everolimus blocca la proliferazione cellulare stimolata dai fattori di crescita e dalle citochine e questo sia a carico delle cellule ematopoietiche che delle cellule non ematopoietiche. Everolimus si lega ad un recettore citosolico (FKBP12) (FKbinding protein), appartenente alla classe delle immunofilline. Il complesso everolimus-FKBP12 interagisce con la sua molecola target, mTOR. Tale interazione si traduce in una potente inibizione del segnale di proliferazione cellulare e della traduzione degli RNA messaggeri richiesti per la progressione del ciclo cellulare dalla fase G1 alla fase S. Everolimus agisce quindi in una fase tardiva del ciclo cellulare attraverso un meccanismo distinto da quello dei CNI. Questo ne giustifica e permette la co-somministrazione.

TABELLA I - DIFFERENZE CHIAVE DI FARMACOCINETICA FRA EVEROLIMUS E SIROLIMUS

\begin{tabular}{|l|c|c|}
\hline & Everolimus & Sirolimus \\
\hline Biodisponibilità orale & $20 \%$ & $14 \%$ \\
\hline $\begin{array}{l}\text { Tempo per raggiungere } \\
\text { Tmax }\end{array}$ & $1-2$ ore & $1-2$ ore \\
\hline Tempo di dimezzamento & 28 ore & 62 ore \\
\hline Intervallo fra le dosi & BID & OD \\
\hline $\begin{array}{l}\text { Tempo per raggiungere } \\
\text { lo steady state }\end{array}$ & 4 giorni & $5-7$ giorni \\
\hline $\begin{array}{l}\text { Legame alle proteine } \\
\text { plasmatiche }\end{array}$ & $74 \%$ & $92 \%$ \\
\hline
\end{tabular}


Poiché everolimus inibisce la crescita cellulare indotta dai fattori di crescita, i suoi effetti antiproliferativi non sono limitati al sistema immunitario; il farmaco interferisce anche con la proliferazione delle cellule muscolari lisce e le cellule endoteliali $(4,5)$. Infine interferisce, e, vedremo l'importanza, con la crescita e la proliferazione delle cellule neoplastiche.

Sono infine da tenere in adeguata considerazione le interazioni farmacologiche di everolimus con altri farmaci. Le principali interazioni sono evidenziate dalla Tabella II (6). Questo è soprattutto importante per le ripercussioni che tali farmaci possono avere sulle anche forti interazioni esistenti fra everolimus e ciclosporina.

\section{Everolimus nel trapianto renale}

Sostanzialmente everolimus nel trapianto di rene viene impiegato in due condizioni:

Trapianto renale de novo. In associazione con CNI al fine di minimizzarne le dosi o addirittura di sospenderle completamente.

Terapia di mantenimento. Si tratta dell'aggiunta di everolimus in pazienti in mantenimento con terapia a base di CNI, al fine di ridurre i dosaggi di questo per prevenire e/o arrestare la disfunzione renale cronica e la nefrotossicità causata da CNI.

\section{Everolimus de novo}

I due studi autorizzativi internazionali sull'everolimus (B251 e B201) sono stati effettuati agli inizi del 2000 (7, 8). Si tratta di due studi con follow-up di tre anni, randomizzati, multicentrici, a gruppi paralleli atti a valutare l'efficienza e la sicurezza di everolimus somministrato

TABELLA II - INTERAZIONI FARMACOLOGICHE DI EVEROLIMUS CON ALTRI FARMACI

\begin{tabular}{|c|c|}
\hline Gruppi di farmaci & Effetti \\
\hline $\begin{array}{l}\text { Inibitori del CYP3A4 e glicoproteina C } \\
\text { Antifungini, antibiotici macrolidi, } \\
\text { calcioantagonisti, inibitori della proteasi }\end{array}$ & $\begin{array}{l}\text { Aumentano le concentrazioni } \\
\text { ematiche di everolimus }\end{array}$ \\
\hline $\begin{array}{c}\text { Ciclosporina (moderato inibitore } \\
\text { del CYP3A4) }\end{array}$ & $\begin{array}{c}\text { Aumenta in maniera significativa } \\
\text { le concentrazioni ematiche di } \\
\text { everolimus }\end{array}$ \\
\hline $\begin{array}{c}\text { Induttori del CYP3A4 } \\
\text { Anticonvulsivanti, farmaci anti HIV }\end{array}$ & $\begin{array}{c}\text { Possono abbassare le } \\
\text { concentrazioni ematiche di } \\
\text { everolimus }\end{array}$ \\
\hline $\begin{array}{c}\text { Rifampicina (forte induttore del } \\
\text { CYP3A4) }\end{array}$ & $\begin{array}{c}\text { Riduce in maniera significativa } \\
\text { le concentrazioni ematiche di } \\
\text { everolimus }\end{array}$ \\
\hline
\end{tabular}

a $1,5 \mathrm{mg} /$ die o $3 \mathrm{mg} /$ die verso il micofenolato mofetile (MMF) a $2 \mathrm{~g} /$ die, nell'ambito di una triplice terapia con CsA (ciclosporina A) e steroidi. Sono stati complessivamente arruolati circa 1200 pazienti nei due studi. La ciclosporina veniva somministrata a dosi standard. In entrambi gli studi l'efficacia di everolimus era paragonabile a quella di MMF.

I messaggi importanti provenienti da entrambi gli studi erano:

- L'efficacia di everolimus era paragonabile a quella di MMF.

- L'efficacia era simile somministrando $1,5 \mathrm{mg} / \mathrm{die}$ o $3 \mathrm{mg} /$ die purché il trough level fosse $>3 \mathrm{ng} / \mathrm{mL}$.

- Il filtrato glomerulare era più elevato nei pazienti in trattamento con MMF.

Quest'ultimo punto ha fatto sospettare un'aumentata nefrotossicità dovuta ad interferenza fra everolimus e ciclosporina somministrata a dosi standard.

Uno studio successivo (B156) (9) ha messo a confronto everolimus con dosi standard o ridotte di ciclosporina. Tutti i pazienti arruolati ricevevano everolimus a $3 \mathrm{mg} /$ die in associazione a steroidi e basiliximab come terapia di induzione. Dopo il trapianto i pazienti erano randomizzati a ricevere dosi piene o ridotte di ciclosporina.

Lo studio ha documentato un basso numero di rigetti in entrambi i bracci e filtrato glomerulare più elevato nel gruppo di pazienti a dose ridotta di ciclosporina. Alla luce di questi risultati, due nuovi studi sono stati effettuati per confrontare dosi di 1,5 o $3 \mathrm{mg} /$ die di everolimus con dosi ridotte di ciclosporina e steroidi (10). In questi sudi è stato introdotto il concetto di monitoraggio terapeutico delle dosi farmacologiche. Le dosi di everolimus erano aggiustate per avere trough levels $>3 \mathrm{ng} /$ $\mathrm{mL}$. Ugualmente erano variate le dosi di ciclosporina. I risultati di questi studi hanno confermato l'efficacia e la sicurezza di everolimus associato a basse dosi di ciclosporina. Un risultato comune a tutti gli studi citati è stata la bassa incidenza di infezioni da CMV (citomegalovirus) nei pazienti in trattamento con everolimus.

Recentemente un nuovo approccio immunosoppressivo è stato quello di associare dosi molto elevate di everolimus a dosi molto ridotte di ciclosporina nellintento di migliorare ulteriormente nel tempo la filtrazione glomerulare. Lo studio Everest (11) ha dimostrato l'efficacia e la sicurezza di dosi molto elevate di everolimus $(\mathrm{C} 0=8-12 \mathrm{ng} /$ $\mathrm{mL}$ ) con dosi molto ridotte di ciclosporina (C2 (concentrazione ematica alla seconda ora) $=150-300)$. I rigetti acuti sono stati adeguatamente bassi, senza avere effetti collaterali di rilievo. Il filtrato glomerulare a un anno, pur elevato, non è stato così significativo come atteso, probabilmente perché molti investigatori non hanno tenuto i livelli di ciclosporina bassi come da protocollo. 
Tale associazione farmacologica ha comunque permesso di ridurre, rispetto ai livelli standard, i livelli di C2 di ciclosporina del $73 \%$.

La Figura 1 rappresenta l'evoluzione nel tempo dei diversi studi citati e gli ottimi risultati ottenuti con una associazione di ciclosporina ed everolimus attentamente controllata.

L'ultima tappa fino ad oggi raggiunta è stata l'eliminazione completa della ciclosporina. Nello studio Zeus (12), è stato possibile a 4 mesi dal trapianto sospendere in un braccio dello studio, completamente la ciclosporina, grazie all'introduzione di acido micofenolico. Con la sospensione completa della ciclosporina, si ottenevano filtrati glomerulari più elevati, senza aumento di rigetti acuti. Il follow-up dello studio Zeus è ora di 3 anni ed i risultati si confermano ottimi.

\section{Everolimus in pazienti in mantenimento con CNI}

Due gruppi di pazienti si avvarrebbero in particolare di conversione terapeutica ad everolimus:

Pazienti con disfunzione renale e documentata nefrotossicità da CNI.

Pazienti che sviluppano neoplasie post-trapianto.

Pazienti con disfunzione renale e documentata nefrotossicità da CNI

Come già detto gli agenti antiproliferativi, fra cui everolimus, permettendo la riduzione dei dosaggi di CNI,

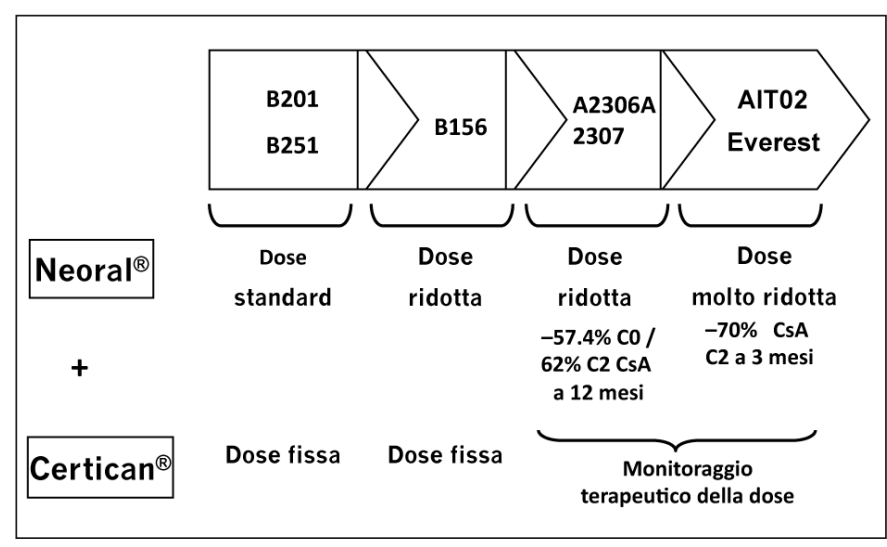

Fig. 1 - Ottimizzazione e tappe cliniche dell'uso di everolimus nel trapianto renale.

limitano la nefrotossicità e il danno renale progressivo tipico della nefropatia cronica da trapianto. Esisterebbe quindi un'indicazione a fare uno switch terapeutico nei pazienti in terapia con CNI, che sviluppano nefrotossicità connessa a tali farmaci.

Lo studio condotto da Cataneo-Dàvila (13) ha valutato la funzione renale in pazienti con nefropatia cronica, dopo la conversione a regimi immunosoppressivi contenenti everolimus, al fine di ridurre o eliminare la somministrazione di CNI. Il dosaggio di CNI è stato ridotto dell' $80 \%$ o interrotto. I risultati a 1 anno di questo studio hanno permesso di concludere che la riduzione o l'eliminazione di CNI e l'introduzione di everolimus possono essere utili per rallentare la progressione del danno renale anche nei pazienti con nefropatia cronica da trapianto.

I risultati dello studio CONVERT (14) permettono di
Fig. 2 - Rapporti fra everolimus e neoplasie.

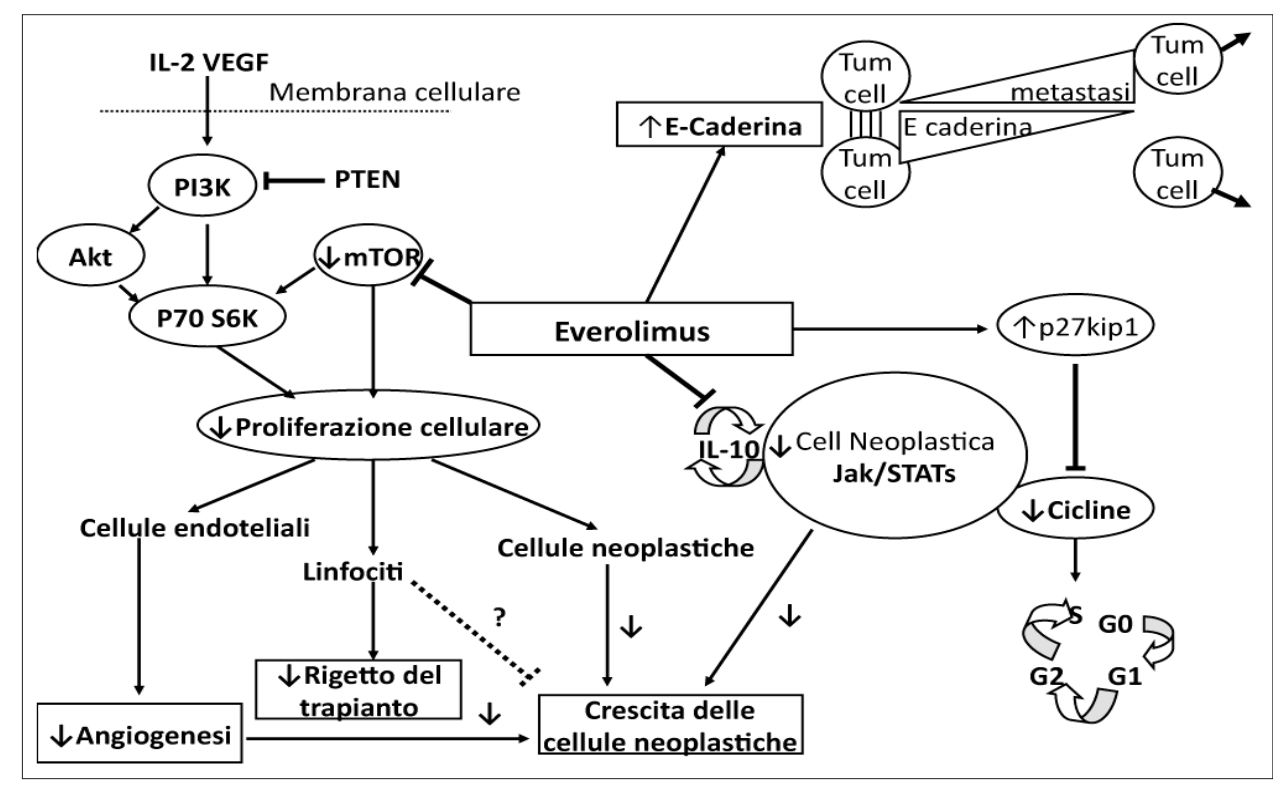


concludere che l'interruzione o la riduzione di CNI con introduzione di inibitori del segnale di proliferazione è però probabilmente inutile, se non di danno, quando il filtrato glomerulare è $<40 \mathrm{ml} / \mathrm{min}$ o quando si abbia una proteinuria $>800 \mathrm{mg} /$ die.

In caso di nefropatia da CNI la conversione deve essere sempre quanto più possibile precoce: è infatti del tutto inefficace attuarla quando il danno renale è già consolidato e la funzionalità renale compromessa.

\section{Pazienti che sviluppano neoplasie post-trapianto}

Il razionale per l'utilizzo dei PSI (inibitori del segnale di proliferazione) in pazienti trapiantati che sviluppano neoplasie risiede, oltre che nelle dimostrate proprietà antiproliferative e antiangiogenetiche (Fig. 2), anche in un diretto coinvolgimento della via di trasduzione mTOR nella progressione di diversi tipi di tumore, tra cui il carcinoma del rene, il sarcoma di Kaposi, i tumori della mammella, del colon, della tiroide, l'epatocarcinoma, i linfomi e i carcinomi cutanei. I PSI sono stati utilizzati con successo in protocolli di conversione in pazienti con carcinoma squamoso o carcinoma basocellulare, e, in casi di sarcoma di Kaposi, la conversione a PSI ha indotto la completa remissione della malattia (15).

In caso quindi di diagnosi di neoplasia post-trapianto, è raccomandabile la minimizzazione o la sospensione dei CNI e l’introduzione di everolimus.

\section{Effetti collaterali dell'impiego di everolimus}

Gli effetti antiproliferativi di everolimus non sono limitati, come già detto, al sistema immunitario. Infatti l'effetto antiproliferativo è dovuto al blocco della serin treonin kinasi

\section{TABELLA III - EFFETTI BENEFICI ED AVVERSI CONNESSI ALL'EFFETTO ANTIPROLIFERATIVO DI EVEROLIMUS}

\section{Effetti benefici}

\section{Effetti indesiderati}

- Effetto antivirale

- Effetto antiaterosclerotico

- Effetto antineoplastico

\section{- Proteinuria}

- Ritardo nella guarigione della ferita

- Linfocele
mTOR che è diffusa in tutte le cellule dell'organismo. Tale effetto antiproliferativo è alla base di effetti collaterali utili, ma anche di effetti collaterali indesiderati (Tab. III).

Everolimus ha un effetto protettivo nei confronti di patologie virali in particolare il CMV. Tutti gli studi citati riportano una incidenza estremamente bassa di infezione citomegalica quando viene somministrato everolimus. Questo è particolarmente importante, poiché la malattia citomegalica è associata a rigetto del trapianto, ridotta sopravvivenza dell'organo e del paziente e predispone allo sviluppo di neoplasie (16).

Gli inibitori del segnale di proliferazione inibiscono la proliferazione delle cellule muscolari lisce e delle cellule endoteliali. In tal modo inibiscono il rimodellamento vascolare. Dati clinici provenienti soprattutto dal trapianto di cuore documentano che gli mTOR inibitori esercitano un effetto protettivo sul cuore e sull'apparato vascolare.

In un trial prospettico, randomizzato, controllato, l'incidenza di nuove neoplasie dopo trapianto renale era più bassa nei pazienti che ricevevano sirolimus rispetto a pazienti che ricevevano altri farmaci immunosoppressivi (17). Questo può essere ascritto al fatto già menzionato dell'attività del farmaco su mTOR che è diffusa in tutte le cellule, incluse le cellule neoplastiche (Fig. 2). Un fatto peculiare degli inibitori di mTOR, incluso everolimus, è il blocco dell'angiogenesi che favorisce l'accrescimento della neoplasia. L'efficacia di everolimus anche al di fuori del campo dei trapianti è stata ben documentata nel carcinoma renale (18) e in altre neoplasie.

Problemi con la guarigione della ferita chirurgica e un aumento di formazione di linfoceli sono stati segnalati con l'uso di inibitori del segnale di proliferazione. Tali effetti sono probabilmente da ricondurre all'effetto antiproliferativo esercitato sui fibroblasti. Per limitare tali problemi è opportuno non trattare pazienti obesi con everolimus, non usare mai dose di carico del farmaco, adeguare sempre il dosaggio del farmaco ed evitare o minimizzare l'uso degli steroidi. Presenza di proteinuria è stata descritta in associazione con l'uso di inibitori del segnale di proliferazione. Una recente analisi di proteomica associata a biopsie protocollari ha permesso di attribuire la proteinuria all'effetto antiproliferativo e proapoptotico dell'everolimus. La proteinuria è prevalentemente tubulare e associata ad aumentata apoptosi di cellule tubulari e di podociti (19). La proteinuria in genere appare nel primo periodo post-trapianto e scompare col tempo senza compromettere la funzione renale (20). Infine l'uso di inibitori di mTOR è associato a maggiore incidenza di necrosi tubulare, che risulta anche più protratta, derivante da danno di ischemia riperfusione. Si tratta ancora di effetto antiproliferativo esercitato sulle cellule tubulari. Il fenomeno è più marcato con il sirolimus, probabilmente per la necessità di dare una dose carico per raggiungere più rapidamente lo steady state (21). 


\section{Conclusioni}

In conclusione l'everolimus si caratterizza per una particolare farmacocinetica che lo rende il farmaco di prima scelta, nell'ambito degli inibitori del segnale di proliferazione.

Il suo impiego de novo, nell'immunosoppressione post-trapianto, trova indicazione in protocolli terapeutici che prevedono la minimizzazione degli inibitori della calcineurina. In particolare possono avvalersene riceventi di reni marginali, pazienti a rischio cardiovascolare, pazienti a rischio per infezione da CMV e pazienti con anamnesi pregressa per neoplasie.

L'impiego di everolimus è indicato anche in pazienti in terapia di mantenimento con inibitori della calcineurina. In particolare nei pazienti che sviluppano nefrotossicità da connettersi all'impiego di tali farmaci. Anche pazienti in terapia di mantenimento che sviluppano neoplasie post-trapianto si avvalgono di un farmaco come l'everolimus che ha documentato effetto antineoplastico anche a prescindere dall'effetto immunosoppressivo.

È opportuno sottolineare l'interferenza di everolimus con CNI. Questo può con facilità causare nefrotossicità, per cui è opportuno un frequente dosaggio e adeguamento posologico dei farmaci.

Gli effetti collaterali indesiderabili sono controllabili in modo relativamente semplice.

Ritardo di guarigione della ferita chirurgica, linfoceli e ritardata ripresa della funzione renale sono meno frequenti con everolimus rispetto a sirolimus, probabilmente perché con quest'ultimo farmaco è necessario somministrare all'inizio una dose carico. $\grave{E}$ anche opportuno prendere in considerazione protocolli terapeutici che prevedano riduzione rapida o sospensione degli steroidi. E anche possibile ritardare l'immissione degli inibitori del mTOR, avvalendosi di dosi piene di CNI, solo nel primissimo periodo post-trapianto. La proteinuria infine, che in un primo momento aveva destato preoccupazione, col protrarsi degli studi ha mostrato essere fenomeno relativamente occasionale, relativo al primo periodo e connesso all'effetto proapoptotico. Nel lungo termine la proteinuria tende a scomparire e non determina deterioramento della funzione renale.

\author{
Indirizzo degli Autori: \\ Maurizio Salvadori, MD \\ Renal Unit \\ Azienda Ospedaliera Careggi \\ Viale Pieraccini 16 \\ 50139 Firenze \\ maurizio.salvadori@unifi.it
}

\section{Bibliografia}

1. Gjertson DW. Survival trends in long-term first cadaverdonor kidney transplants. Clin Transpl 1991: 225-35.

2. Augustine JJ, Bodziak KA, Hricik DE. Use of sirolimus in solid organ transplantation Drugs 2007; 67: 369-91.

3. MacDonald AS. Use of mTOR inhibitors in human organ transplantation. Exper Rev Clin Immunol 2007; 3: 423-36.

4. Schuurmann HJ, Ringers J, Schuler W, et al. Oral efficacy of the macrolide immunosuppressant SDZ-RAD and of cyclosporine microemulsion in cynomolgus monkey kidney transplantation. Transplantation 2000; 69: 737-42.

5. Cole OJ, Shehata M, Rigg KM. Effect of SDZ RAD on transplant arteriosclerosis in the rat aortic model. Transplant Proc 1998; 30: 2200-03.

6. Kovarik JM, Beyer D, Schmouder RL. Everolimus drug interactions: application of a classification system for cli- nical decision making. Biopharm Drug Dispos 2006; 27: 421-6.

7. Vitko S, Margreiter R, Weimar W, et al. Three-year efficacy and safety results from a study of everolimus versus mycophenolate mofetil in de novo renal transplant patients. Am J Transplant 2005; 5 (10): 2521-30.

8. Lorber MI, Mulgaonkar S, Butt KM, et al. Everolimus versus mycophenolate mofetil in the prevention of rejection in de novo renal transplant recipients: a 3-year randomized, multicenter, phase III study. Transplantation 2005; 80 (2): 244-52.

9. Nashan B, Curtis J, Ponticelli C, Mourad G, Jaffe J, Haas T; 156 Study Group. Everolimus and reduced-exposure cyclosporine in de novo renal-transplant recipients: a three-year phase II, randomized, multicenter, open label study. Transplantation 2004; 78: 1332-40.

10. Vitko S, Tedesco H, Eris J, et al. Everolimus with opti- 
mized cyclosporine dosing in renal transplant recipients: 6-month safety and efficacy results of two randomized studies. Am J Transplant 2004; 4 (4): 626-35.

11. Salvadori M, Scolari MP, Bertoni E, et al. Everolimus with very low-exposure Cyclosporine A in de novo kidney transplantation: a multicenter, randomized, controlled trial. Transplantation 2009; 88 (10): 1194-202.

12. Budde K, Becker T, Arns W,et al. Everolimus-based, calcineurin-inhibitor-free regimen in recipients of de-novo kidney transplants: an open-label, randomised, controlled trial. Lancet 2011 epub ahead of prints.

13. Cataneo-Davila A, Zuniga-Varga J, Correa-Rotter R, et al. Renal function outcomes in kidney transplant recipients after conversion to everolimus-based immunosuppression regimen with CNI reduction or elimination. Transplant Proc 2009; 41: 4138-46.

14. Schena FP, Pascoe MD, Alberu J, et al. Sirolimus CONVERT Trial Study Group. Conversion from calcineurin inhibitors to sirolimus maintenance therapy in renal allograft recipients: 24-month efficacy and safety from the CONVERT trial. Transplantation 2009; 87: 233-42.

15. Valantine $H$. Is there a role for proliferation signal/mTOR inhibitors in the prevention and treatment of de novo malignancies after heart transplantation? Lessons learned from renal transplantation and oncology. J Heart Lung Transplant 2007; 26: 557-64.

16. Fishman JA, Emery V, Freeman R et al. Cytomegalovirus in transplantation- challenging the status quo. Clin Transplant 2007; 21: 149-58.

17. Campistol JM, Eris J, Oberbauer R, et al. Sirolimus therapy after early cyclosporine withdrawal reduces the risk for cancer in adult renal transplantation. J Am Soc Nephrol 2006; 17 (2): 581-9.

18. Motzer RJ, Escudier B, Oudard S, et al. Efficacy of everolimus in advanced renal cell carcinoma: a double-blind, randomized, placebo-controlled phase III trial. Lancet 2008; 372: 449-56.

19. Bertoni E, Bruschi M, Candiano G, et al Post-transplant proteinuria associated with everolimus. Transplant Proc 2009; 41 (4): 216-7.

20. Bertoni E, Larti A, Rosso G, Zanazzi M, Di Maria L, Salvadori M. Good outcomes with Cyclosporine very low exposure with everolimus high exposure in renal transplant patients. J Nephrol 2011, epub ahead of prints.

21. Stallone G, Di Paolo S, Schena A, et al. Addition of sirolimus to cyclosporine delays the recovery from delayed graft function but does not affect 1-year graft function. J Am Soc Nephrol 2004; 15 (1): 228- s33. 